\title{
Kronos Abuses His Son: A Case Study of Severe Interpersonal Trauma, Dissociation, and Survival in Adolescence
}

\author{
Penny Papanikolopoulos \\ TACT HELLAS - Training and Consultation Today in Hellas, Athens, Greece \\ Despina D. Konstas \\ Hellenic American University, Athens, Greece \\ Tessa-Ava Prattos-Spongalides \\ TACT HELLAS - Training and Consultation Today in Hellas, Athens, Greece \\ Maria Belivanaki \\ Gerasimos Kolaitis \\ Department of Child Psychiatry, Medical School, National and Kapodistrian University of Athens, \\ "Aghia Sophia" Children's Hospital, Athens, Greece
}

\begin{abstract}
This case study presents case conceptualization, therapeutic intervention, and the subjective and objective therapeutic progress of a 14-year-old adolescent hospitalized with posttraumatic stress disorder (PTSD) following emotional, physical, and sexual abuse by his father. The adaptive information processing (AIP) model that informs eye movement desensitization and reprocessing (EMDR) therapy and the theory of structural dissociation of the personality (TSDP) were used to conceptualize and guide the treatment. Stabilization and orientation to the present were essential to integrate his traumatic memories into a life narrative, and this became a major goal and an outcome of treatment. A single-case AB design was applied in assessing the impact of intervention. The UCLA PTSD Symptom Scale, Strengths and Difficulties Questionnaire-HEL (SDQ-HEL), State-Trait Anxiety Inventory (STAI), and Dissociative Experiences Scale II were administered at 5 different time points to assess changes in the youth's subjective emotional state and indicated substantial improvement. In addition, objective behavior change (using O'Neill's Behavior Checklist) was recorded on a daily basis for 7 months and showed a large decrease in the frequency of targeted maladaptive behaviors. The article describes the treatment process which helped the youth to regain a sense of time; establish a coherent sense of self; and maintain adaptive perceptions, emotions, attitudes, and behaviors.
\end{abstract}

Keywords: posttraumatic stress disorder (PTSD); eye movement desensitization and reprocessing (EMDR); adaptive information processing model (AIP); theory of structural dissociation of the personality (TSDP); dissociation; childhood and adolescence

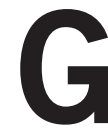
reek mythology tells us that the Titan Kronos, chose Rhea as his wife and she gave birth to the gods, who were prophesized to overthrow him. Kronos then swallowed his children for fear that this prophecy might be realized. The word Kronos is also very close to the Greek word chronos which means time. Time, as in past, present, and future, is an essential element of this case. This adolescent, abused by his father, appeared trapped with a disoriented sense of time on occasion. At initial presentation, he was confused and his experiences were extremely disconnected. There were times when he was triggered in the present and would relive his trauma. It took several months and numerous therapy sessions before he was able to address his life story in a chronological order.

Eye movement desensitization and reprocessing (EMDR) therapy, guided by the adaptive information processing (AIP) model (F. Shapiro, 2001), was applied, whereas the theory of structural dissociation 
of personality (TSDP; Van der Hart, Nijenhuis, \& Steele, 2006) was used for conceptual understanding of the case. This case study describes the efforts that were made to help this adolescent regain a sense of time; establish a coherent sense of self; and maintain a more adaptive resolution in terms of perceptions, emotions, attitudes, and behaviors.

\section{Literature Review}

\section{Trauma and Dissociation in Posttraumatic Stress Disorder}

The traumatizing events associated with posttraumatic stress disorder (PTSD) may be a single trauma such as an accident or may range all the way to the severe early developmental traumatization of a helpless child. Needless to say, not all severe and impactful developmental trauma are contained within the definition of PTSD. Nonetheless, childhood traumatization interferes with the child's development of a cohesive and coherent personality structure. According to Main and Morgan (as cited in Van der Hart, Nijenhuis, \& Steele, 2005), chronic childhood abuse can compromise the integrative capacity and the development of secure attachment and caretaker abuse may lead to the development of disorganized attachment in the child. This type of attachment is strongly linked to dissociation (e.g., Carlson; Ogawa, Sroufe, Weinfeld, Carlson, \& Egeland, as cited in Van der Hart et al., 2005).

As trauma therapists, we may think of dissociation as a way of reacting to and organizing information occurring at the time of the trauma. From this perspective, dissociation can be viewed as an adaptive response to physical and/or sexual abuse because it may decrease the overwhelming distress created by trauma and by the conflicting and demanding environment in which the child is living (Knipe, 2014). However, even though dissociation may help children survive and cope with trauma, it can be considered maladaptive because it inhibits appropriate learning and healthy attachment and tends to persist over time. More specifically, TSDP views trauma-generated dissociation of the personality primarily as an integrative failure-a "falling apart." Subsequently, it may have some limited adaptive value in that one (or more) part, emotional part of the personality (EP), keeps the traumatic memories, and one (or more) part, apparently normal part of the personality (ANP), is able to function, to some degree, in daily life (Van der Hart et al., 2006). If dissociation of the personality continues into adulthood, when the original danger no longer exists, it will most likely continue to be maladaptive
(International Society for the Study of Trauma and Dissociation [ISSTD], 2016).

\section{Treatment of Trauma and Dissociation in Posttraumatic Stress Disorder}

The ISSTD Task Force for Children and Adolescents has developed guidelines for the assessment and treatment of dissociative symptoms in traumatized children and adolescents. To foster the coherence of self and promote healthy adaptation according to the child or adolescent's ability to enter a relationship and feel safe again in the present (Van der Hart, Groenendijk, Gonzalez, Mosquera, \& Solomon, 2014), the guidelines recommend proceeding slowly by titrating therapeutic interventions while maintaining safety, building trust, and using a phased approach (ISSTD, 2004). The three treatment phases of Pierre Janet (as cited in Van der Hart et al., 2005) are History, Assessment, Stabilization, Symptom Reduction, and Skills Building (Phase I); Treatment of Traumatic Memories (Phase II); and Personality Reintegration and Rehabilitation (Phase III). The more complex the trauma is, the less likely that these phases will be applied in order. Backtracking to Phase I is necessary in order that safety and trust are promoted in the clienttherapist relationship (Van der Hart et al., 2014).

\section{The Adaptive Information Processing Model and EMDR Therapy}

EMDR therapy is an empirically valid treatment for PTSD, based on numerous randomized controlled trials and several meta-analyses (e.g., Chen, Zhang, Hu, \& Liang, 2015). There is also preliminary evidence from several controlled trials and published case reports for EMDR's effectiveness with other presenting problems, such as depression (e.g., Gauhar, 2016) and anxiety disorders (e.g., Faretta, 2013), following difficult life events. EMDR is one of two psychotherapies recommended by the World Health Organization (2013) for children, adolescents, and adults with PTSD.

The EMDR Therapy Standard Protocol has eight specific phases. Each phase has a purpose and procedure. The phases are as follows: Phase 1, History taking and building the therapeutic alliance. This phase provides an opportunity to identify salient issues (past, present, and future). AIP is used for case conceptualization and a three-pronged treatment plan. Not all clients are able to give a clear history at onset of therapy. This phase is also useful for assessing client appropriateness for EMDR therapy. Phase 2, Client stabilization and preparation. During this phase, general assessment for a sense of self-control and 
attachment issues continues while the therapist psychoeducates the client to the particular therapy. Phase 3, Assessment activation of traumatic memory network. The therapist elicits the image, negative cognition, currently desired positive cognition, current emotion, physical sensation, and baseline pertaining to particular target. Phase 4, Desensitization of traumatic memory with the use of bilateral stimulation up to adaptive resolution while monitoring distress on the level of Subjective Units of Disturbance (SUD) scale, where $0=$ no disturbance and $10=$ worst possible. In this phase, the client focuses on aspects of the targeted memory, while engaging in dual attention (DA). This allows the client to be in the present while mentally accessing a traumatic event from the past. In some ways, DA helps the client to modulate self between past and present without losing self in-the-past trauma (Gonzalez \& Mosquera, 2012). It is desirable to process past, present, future targets and associated channels: image, cognitions, emotions, and somatic sensations. There is a use of cognitive interweaves when processed blocking occurs. Phase 5, Installation allows an increase of connections and generalizations to positive cognitive networks. The therapist and client identify the most appropriate positive cognition and reinforce validity of positive belief. The validity of the positive cognition should be a 6 or 7 on the Validity of Cognition (VOC) scale, where $1=$ completely false and $7=$ totally true. Phase 6 , Body scanning is used to monitor and clear any residual disturbing feelings in the body. A neutral body response is the goal; otherwise, desensitization will continue. Phase 7 , Closure ensures client stability at the end of an EMDR session and between incomplete sessions. There is possible use of self-help techniques and self-observation between sessions. Phase 8, Reevaluation takes place at the beginning of the next session, and it assesses treatment effects. Therapist and client explore what has emerged since last session and access memory and its current level of disturbance.

EMDR therapy is guided by the AIP model which seeks to explain pathology, predict clinical outcomes, and guide case conceptualization and treatment procedures in EMDR therapy (F. Shapiro, 2001; F. Shapiro \& Solomon, 2008). It is posited that when the natural AIP system is disrupted, unresolved experiences can leave behind a residue in state specific form (F. Shapiro, 2007). Earlier adverse life experiences are understood to create a template for later psychological problems. The AIP model posits that for every problem there are past, present, and future aspects: Past distressing memories, present-day triggers that usually bring the client in for help, and future challenges where the client may be concerned about what may occur or go wrong. EMDR reprocessing is thought to bring about adaptive resolution by removing blocks and allowing the reintegration of memory to a more holistic adaptive resolution of perceptions, attitudes, emotions, and body sensations. EMDR therapy appears to restimulate the AIP self-healing system.

Similarly to ISSTD's recommendations, Shapiro also advises practitioners to be flexible when providing EMDR to clients who have difficulties in attaining affect tolerance, modulation, and self-regulation. She recommends that clinicians be aware of possible dissociation and show clinical judgment concerning the timing of traumatic memory reprocessing during treatment (F. Shapiro, 2001).

\section{Theory of Structural Dissociation of the Personality}

The TSDP provides useful concepts to explain the impact of trauma on the personality of an abuse survivor. One principle of TSDP is that each human being has an integrative capacity to deal with experiences. The integrative capacity entails two major mental actions, namely, synthesis and realization. Synthesis can be thought of as the way one perceives, compares, differentiates, and/or categorizes internal and external experiences in the present and over time. Realization involves higher level mental actions that entail awareness of reality, accepting it, and adapting to it: It entails (a) personification, that is, knowing "this is what happened to me" and knowing my feelings and thoughts about it and (b) presentification, that is, being grounded in the present while able to integrate the past, the present, and the expected future (Van der Hart et al., 2006).

Thus, integration at the level of realization can be thought of as staying in the present while describing a past whole life narrative and owning experience. The person can express and feel his or her painful experience and memories without avoiding them. This causes a shift in his or her view of self and the internal phobias once experienced because of the trauma and/or memories. According to TSDP, the failure to integrate traumatic experiences basically yields a structural dissociation of the personality into two or more mental systems, labeled as dissociative parts of the personality (Van der Hart et al., 2006). There are moments when the individual appears to relive the trauma and other occasions when the individual presents with a lack of personification or a sense of "not me" regarding one's own body sensations and feelings. There also appears to be a sense of "not real" as opposed to knowing one's story and seeing options 
for a future while having a stable sense of self and what has occurred in the past.

This structural dissociation of the personality involves a division between at least one EP that is essentially associated with reexperiencing the trauma and at least one ANP that has failed to integrate the traumatic experience and the involved EP and that engages in matters of daily life. These "structural" parts are not static, they are dynamic interrelated parts which are not fully aware of each other and often not in cooperation with each other. The more intense the traumatization, the earlier it starts in life and the longer it lasts, probably the more complex the dissociation of the personality becomes (Van der Hart et al., 2006).

\section{Method}

\section{Procedures}

The current project entails a case study of a 14-yearold boy, with a diagnosis of PTSD who endured emotional, physical, and sexual abuse by his father, and who presented with severe emotional and behavioral problems and dissociative symptoms. Two clinicians provided EMDR therapy, guided by the AIP model and the TSDP. Treatment was provided over a 7-month period, while the boy was an inpatient in a secure university hospital psychiatric unit for children and adolescents. Applying a single-case AB design, several instruments and measures were employed to assess changes in the youth's subjective emotional states over time. In addition, objective behavior change was also recorded over time on the hospital unit where he resided. Goals of EMDR treatment were to help this adolescent regain a sense of time and a coherent sense of self; establish and maintain a more adaptive resolution of perceptions, emotions, attitudes and behaviors; and integrate the traumatic memories into a life narrative.

\section{Treatment}

Treatment in the Hospital Setting. The participant, "Stephanos" (alias), was an inpatient on the child and adolescent psychiatric unit for 7 months. Although the unit was a short-stay unit, the staff of the department did everything they could administratively to keep him there so that he would have ample time for his therapy. The structured hospital program provided a stable and caring environment. The program included daily community meetings between the inpatient children and adolescents and the staff where feelings and thoughts were shared. Educational programs were provided on an individual basis or small group tutoring sessions. The inpatients attended a weekly support group led by mental health staff. Stephanos's behavior and medication changes were monitored by a child psychiatrist on the ward. Finally, once or twice a week, Stephanos was allowed a social outing outside the hospital.

EMDR Therapy. Two licensed psychologists, EMDR Europe-accredited practitioners, consultants, and external colleagues of the university hospital, with more than 30 years of clinical experience were asked to assess as to whether the youth would benefit from EMDR therapy. EMDR was provided as a pro bono treatment over the course of 7 months and included 24 weekly sessions for 2 hours each in the hospital setting. The EMDR approach used for working with this adolescent was two pronged. The first goal was stabilization, to help manage and improve his maladaptive behavior. The second goal was to provide trauma therapy for the multiple abuses he had suffered.

\section{Assessment}

A battery of assessment tools was administered by the EMDR therapists throughout treatment to obtain objective measures of the youth's progress over time. With the exception of the O'Neill Functional Assessment Form and the drawings, the assessment tools were administered on the following dates: Time $1=$ baseline; Time $2=2$ months later; Time $3=4.5$ months later; Time $4=6$ months later; and Time $5=7$ months later. Time 1 was considered baseline because this assessment was completed prior to the beginning of treatment.

The O'Neill Functional Assessment Form. The O’Neill Functional Assessment Form (O’Neill et al., 1997) is a measure of the frequency of behavior over time. This tool was administered by hospital staff for 7 months. It was used daily to record the frequency of target behavior (or lack thereof), its antecedents, consequences, and actual consequences over time. In this case, the following behaviors were identified for observation and recording: (a) hitting, (b) destroying, and (c) yelling/fighting.

Drawings. The therapists engaged the youth in drawings at several points in treatment to view the development and differentiation in his emotional expression as well as the quality of his cognitions. Drawings were used as a subjective measure of progress and were initiated and collected on the following dates: (a) 4 months after treatment onset, (b) 6 months after treatment onset, (c) at the end of treatment, and (d) 11 months after treatment termination. Prior to each drawing, the following instructions were given: Stephanos was always 
instructed to draw himself. Once given the opportunity to draw, the following questions were provided to encourage him further: "How do you feel, what are you thinking, and what are you doing in the drawing?"

UCLA PTSD Symptom Scale. The UCLA PTSD Symptom Scale (Steinberg et al., 2013) is a brief selfreport containing 22 items appropriate for screening for trauma exposure and Diagnostic and Statistical Manual of Mental Disorders (4th ed., DSM-IV) PTSD symptoms in children and adolescents 7-17 years of age. It specifically assesses PTSD symptoms and associated features asking the child to rate the frequency of symptoms in the past month using a Likert scale ranging from 0 indicating a frequency of none, to 4 indicating most. This scale provides a PTSD Reaction Index (PTSD-RI) score which ranges from 0 to 68 with a PTSD-RI score equal to or greater than 38 indicative of likely PTSD. The PTSD-RI total scale displayed good to excellent internal consistency reliability across age ranges, sex, and racial/ ethnic groups ( $\alpha=.88-.91$; Steinberg et al., 2013).

Strengths and Difficulties Questionnaire-HEL. The Strengths and Difficulties Questionnaire-HEL (SDQHEL; Goodman, 1997) is a tool widely used as a brief screening measure of emotional and behavioral difficulties in children and adolescents. The psychometric properties of the SDQ are generally found to be satisfactory (Goodman, 2001) including its Greek version (Giannakopoulos et al., 2013). It consists of 25 items that may be categorized into 5 scales of 5 items each. A Likert scale accompanies each of the 25 items asking the respondent to indicate whether the symptom described in the item is not true or 0 to certainly true or 2. Scoring for each item varies with the item. The SDQ provides a total sum score of all the scales (except the Prosocial scale), namely, the Total Difficulties Score. The Total Difficulties Score ranges from 0 to 40 and is counted as missing if one of the four composite scores are missing. A score of 17-40 indicates high substantial risk of clinically significant problems.

Screen for Childhood Anxiety Related Emotional Disorders-Short Form. The Screen for Childhood Anxiety Related Emotional Disorders-Short Form (SCARED-SF; Birmaher et al., 1999) is a reduced version of the original SCARED. It consists of five items scored $0-2$. This reduced version of the SCARED showed similar psychometrics as the complete SCARED, which is a reliable and valid instrument used to screen for childhood anxiety disorders in clinical settings (Birmaher et al., 1999). Scores typically range from 0 to 10 , with higher scores indicating greater anxiety.
State-Trait Anxiety Inventory. The State-Trait Anxiety Inventory (STAI; Spielberger, Gorsuch, Lushene, Vagg, \& Jacobs, 1983) is a questionnaire administered to measure the intensity at which the client experiences feelings of anxiety. It measures both State and Trait Anxiety (20 questions each); however, for this study, the State Anxiety (S-anxiety) Inventory was administered (i.e., 20 questions). S-anxiety is defined as a type of anxiety that is experienced by a person at the time of a perceived threat and is considered temporary (Spielberger et al., 1983). Internal consistency coefficients for the scale have ranged from .86 to .95 ; test-retest reliability coefficients have ranged from .65 to .75 over a 2-month interval (Spielberger et al., 1983). Considerable evidence attests to the construct and concurrent validity of the scale (Liakos \& Giannitsi, 1984; Spielberger, 1989). The person is asked to indicate how intense the anxiety is on a 4-point Likert scale where 1 is not at all and a 4 is very much so. The scores range from 20 to 80 with higher scores indicative of a greater experience of state anxiety (Spielberger et al., 1983).

Oslo 3-Item Social Support Scale. The Oslo 3-Item Social Support Scale (OSS-3; Dalgard, 1996) consists of three items designed to measure one's level of perceived social support. This scale contains one question about the number of people who can provide a sense of security and support to the individual and two questions about emotional and instrumental support from those people. The total score is calculated by summarizing those three items ranging from 0 to 11 , with values less than 6 identified as indicators of "poor social support."

Impact of Event Scale-Revised. The Impact of Event Scale-Revised (IES-R; Weiss \& Marmar, 1995) consists of 22 items designed to measure the effects of routine life stress, daily traumas, and acute stress. The items describe difficulties a person may experience after a stressful life event. The individual is asked to indicate on a 4-point Likert scale, with a 0 indicating not at all and a 4 indicating extremely, how distressing each of the difficulties named in the items has been in the past week. Scores range from 0 to 88 with scores above 24 indicative of PTSD symptomatology.

Dissociative Experiences Scale II. The Dissociative Experiences Scale II (DES-II; Carlson \& Putnam, 1993) consists of 28 self-report items and is used as a screening instrument for dissociative disorders. This brief measure provides information on the daily frequency of dissociative experiences as well as information on the quality of those experiences as each item is rated on a $0 \%$ (never) to $100 \%$ (always) scale. Results of studies 
of reliability of the DES show good test-retest reliability and internal reliability. Scoring the DES requires totaling the client's responses to the items and dividing that total by 28 (thus, a mean score). The mean score may range from 0 to 100 ; however, a score of 30 or more indicates experiences of dissociation that require follow-up and further investigation.

\section{Description of the Case}

\section{Patient History}

Stephanos was a 14-year-old adolescent admitted and hospitalized in a University Child and Adolescent Psychiatry Department in Greece, because of his aggressive acting out behavior in the therapeutic residential setting where he lived. Based on his history, which was obtained from the file on record at the hospital, he had been abandoned by his mother at birth and raised by his father and paternal grandmother. Although the recorded history was very minimal with large informational gaps related to frequent discontinuation of service, it indicated that he had been neglected and severely beaten by his father from the age 5 years or younger. His father was a substance abuser involved in criminal behavior.

The file mentioned that Stephanos had first entered a state institution at age 3 years. He was in and out of institutions until he was finally taken away from his father at the age of 8 years and placed into state custody. Stephanos had endured severe beatings by his father. During one such incident, Stephanos lost one testicle after being beaten and left on the roof in a rain storm tied to an antenna to die.

Stephanos was frequently moved from one institution to the next, after getting involved in arguments and physical altercations with the staff and other residents. He had noticeable scars on one leg, which he described as the result of being attacked and stabbed in one of these institutions.

\section{Presenting Problems and Diagnosis}

Mental health professionals had given Stephanos several different diagnoses over the years ranging from psychosis, intellectual and developmental disorder, bipolar disorder, attention-deficit/hyperactivity disorder, and oppositional defiant disorder. When Stephanos was first admitted to the University Department, he was on three antipsychotic agents, an antiparkinsonian drug, and a benzodiazepine. At that time, his diagnosis was changed to PTSD by the child psychiatrist working on the inpatient unit team.

Stephanos's specific presenting symptoms were reported by hospital staff as aggressive bursts of anger and rage, fighting with support staff (yelling, hitting, and destroying materials), highly aroused and fearful behavior, with disorganized thinking in moments of high arousal and possible dissociative states which were observed by the child psychiatrist in charge. These dissociative states were characterized by fear, shame, rage, and chaotic emotional states with regressed behaviors (shying away in a corner and being afraid to look at therapist). As explained in the "Conceptualization" section, understanding these states as EPs with evident regressed behavior triggered in the present was helpful in understanding Stephanos's present condition.

\section{Treatment Goals}

The EMDR approach used for working with this adolescent was two pronged. The first goal was stabilization, helping Stephanos to manage and improve his maladaptive behavior and to reduce his difficult and unmanageable behaviors on his unit. Therapeutic goals included decreasing his arousal while simultaneously increasing his grounding in the present, his safety, and his ability to build trust and learn more daily living skills and skills for dealing with interpersonal conflict and internal arousal. To achieve these goals, it was essential for the therapists to understand and identify Stephanos's optimal level of arousal and affect tolerance.

The second goal was to provide trauma therapy for the multiple abuses he had suffered. The therapists were there to understand what had happened to Stephanos and to treat his PTSD with EMDR therapy. The adolescent's life situation was seen through the prism of trauma informed EMDR therapy (i.e., AIP model and the concepts of TSDP). Perhaps, the most important questions that the therapists needed to answer were "What has happened to Stephanos? How did Stephanos survive these events? How did these methods of survival become his coping strategies today?"

\section{Case Conceptualization}

This case entailed early interpersonal trauma and institutional retraumatization. It was seen through the prism of trauma-informed EMDR therapy, using the AIP model and the TSDP (Farrell, 2015) with the past, present, and future orientation and its repetitive traumatic reenactment (Figure 1).

Stephanos's current behaviors appeared clearly linked to his traumatic history, and his present-day reactions were understood as behaviors he had acquired to survive his early severe physical and emotional abuse. Thus, it appeared that some memories were held by dissociated EPs and these memories were conceptualized as 
Phase I:

Hx, Present triggers, Future anxieties/Stabilization/

Symptom Reduction / Skills Building

Premorbid Hx: Attachment

$\mathrm{hx}$, ACE issues,

Institutionalization,

Re-traumatization

$\downarrow$

Trigger Events

Resemblance with father \& rain

$\checkmark$

Stabilization/Symptom Reduction/Skills Bldg

ANP with its defenses, EPs and functionality, Safety, Mindfulness

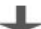

Phase II:

Target Memory /ries - Treatment of Traumatic Memory (EMDR 3-8)

Image or Acoustic, Cognitions, Emotions, Body Sensations

$\downarrow$

Phase III:

Integration of TMs into Life Narrative and Continued Rehabilitation

$\downarrow$

Additional Factors:

Hospital Rules, New Unknown Institutional Placement / Uncertainty

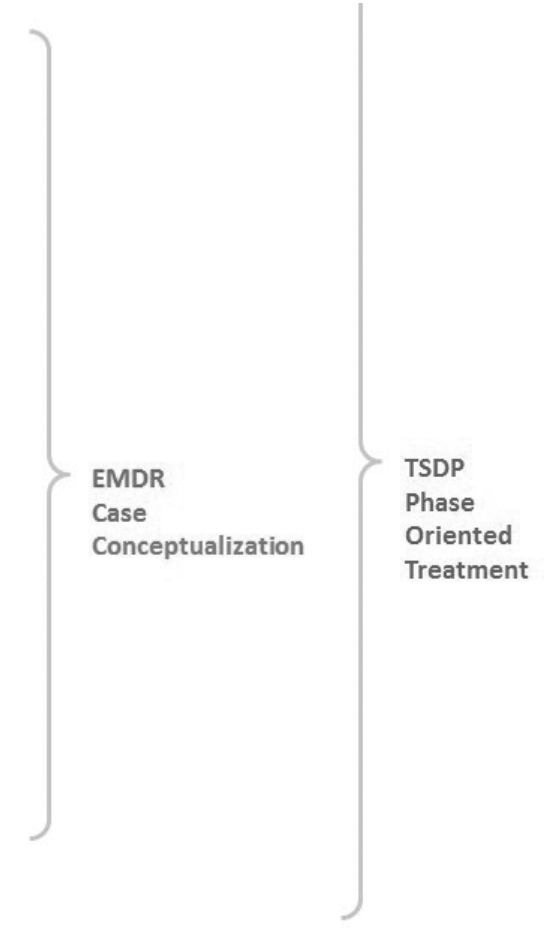

D. Farrell, 2015 - EMDR Conceptualization P. Papanikolopoulos, 2015- TSDP Conceptualization

FIGURE 1. Case conceptualization. Hx = history; ACE = adverse childhood experiences; ANP = apparently normal part of the personality; EP = emotional part of the personality; TM = traumatic memory; TSDP = theory of structural dissociation of the personality. (Modified from Farrell, D. [2015]. EMDR HAP training manual and case conceptualization. Watsonville, CA: EMDR Institute.)

intrusions from an EP into an ANP. For example, when it rained, he would be triggered into an overwhelming physiological panic reaction because he had been beaten and tied on the roof and left to die. Whenever he would see a male that resembled his father on the hospital grounds, he would react with terror. Many times, when staff requested or demanded something from him, he reacted impulsively with a fight response.

It was clear that the dysfunctionally stored memories from early life had been disorganized and disconnected. Stephanos exhibited qualities such as joking, laughter, horseplay initially when we would try to touch on any of his traumatizing life events. Therefore, it seemed that he exhibited positive affect that served the purpose of avoiding prior life trauma. Alternately, he also lowered his head, closed his eyes, and went silent when EPs appeared and the therapists brought them to his attention.

According to Knipe (2014), the extreme avoidance or phobia of the traumatic memories is invested with high levels of dysfunctional positive affect to keep the EPs separate and out of awareness of the ANP and to keep the traumatic memories at bay. There was no doubt that his mental efficiency was weak and disorganized and he had minimal social support in his life, which led him to cling to the few positive relationships in his life (psychiatrists, psychologists, nurses, and one candidate foster mother). Separations from these individuals were very difficult for Stephanos and triggered issues of abandonment and betrayal.

When viewing Stephanos's presentation through the prism of personality dissociation, he had one ANP that was charming, lovable, effective, able to do things, and "look normal" while being oriented to the present for the most part. Nevertheless, Stephanos learned to survive by investing much of his energy in avoiding his traumatic experiences, and this ANP exhibited many defenses. Stephanos overidealized himself as a super strong hero and others as his saviors (physicians, nurses, interns, prospective foster parent). He also exhibited overarousal that originated from EPs that appeared to be reliving trauma in the present. Early in treatment, there were many times where he would be overaroused or disconnected. At times, he tended to be hypergregarious, and at other times, foggy and dissociated.

There were several different EPs. One EP imitated his abusing father's street wise behavior, especially in 
his living quarters on the hospital ward. Another EP was an aggressor which protected him as well as other young children, and which he called "DOPI" (a name found in Internet avatar characters). There was an EP that felt betrayed and another that carried the beatings, the fear, and terror. He introduced another EP named "RAVA" (another name found in Internet avatar characters) which was most interested in revenge and the punishment of his perpetrator and others around him. These dissociative parts (ANP and its defenses and multiple EPs) appeared to be somewhat separated and unaware of each other. Stephanos's personality had a unique structure that had developed as an adaptation to his childhood environment, even though threats were no longer present in his current situation (Knipe, 2014).

\section{EMDR Treatment Progress}

Phase I-History, Initial Assessment, Stabilization, Symptom Reduction, and Skills Building (EMDR Therapy Phases 1-2). The work with Stephanos involved a great deal of preparation, psychoeducation, and encouragement of self-care. Stabilization was one of the initial goals of the therapeutic intervention. A core element was building a relationship characterized by honesty, sincerity, genuine interest, and acceptance of him as a whole. This trust, attunement, and rapport with him was further accomplished by being consistent, patient, and understanding of his difficulties.

Several strategies were used to accomplish stabilization: (a) teaching him to use the four elements exercise (E. Shapiro, 2007) and the Butterfly hug (Artigas, Jarero, Mauer, López Cano, \& Alcalá, 2000) to calm himself down; (b) focusing on breathing work and continuous safety and orientation to the present; (c) grounding and mindfulness exercises; (d) containment exercises; (e) emotional regulation recognizing bodily sensations corresponding to emotional states, thus creating a normalization of thesestates(decreasingfear);(f)identifying his resources and continuously reminding Stephanos of all resources (practical skills, relationships, physical appearance, etc. available to him); (g) treating him as a whole and helping him understand the side of him that was aggressive; and (h) fostering self-compassion and getting his ANP to care for itself and the EPs; (i) the "conference table" technique used to keep the EPs cooperative and in the present (Gonzalez \& Mosquera; Van der Hart et al., as cited in Van der Hart, Groenendijk, Gonzalez, Mosquera, \& Solomon, 2013). The therapists' nonjudgmental stance, continuity, and reliability in the therapeutic relationship characterized this stage of stabilization and psychoeducation. The therapists were attuned to Stephanos as a whole human being.
Working with Stephanos was complicated by the amount of time disorientation; EPs relived events from the past without enough dual awareness to integrate the traumatic material. He exhibited high arousal and was unable to stabilize relatively quickly. He had present triggering events (rain, anyone resembling his father and anyone placing demands on him) as precipitating factors for an EP to come forth. The possibility that his father could approach the hospital grounds was also enough to send him into regressed and terrorized behavior. It was evident that these were examples of his posttraumatic intrusions from EPs into his ANP. There was a need for symptom reduction, skills building, and stabilization. The therapists consistently focused on stabilization and orientation to the present so that Stephanos could understand that the memories were in the past and that he was not presently in danger (Martin, 2016).

When asked to identify a safe calm place, Stephanos spontaneously chose his own heart. He found solace and peace in his heart. Listening to his own calming heartbeat was his "safe state" (O’Shea, 2009). Following development of the safe state, mindfulness exercises were used for connection of heart, mind, and breathing. Stephanos's heart was an imaginary resource to filter all arousal and disturbance (Childre $\&$ Rozman, 2007).

Over the course of therapy, Stephanos was able to allow himself to feel fear, anger, shame, and other intense emotions and thoughts concerning his past and present. He gradually stopped throwing furniture, hitting the walls with his fists, and arguing with staff and other patients. His reactions were much more adaptive as a result of stabilization, heightened awareness of emotions, thoughts, behaviors, as well as connecting to all his resources. He was able to understand the connection of his traumatic past and his present behaviors. He gradually became more attuned and oriented to time. He acquired dual awareness and was able to distinguish past emotional experiences and states from the present.

Phase II-Treatment of Traumatic Memories (EMDR Therapy Phases 3-8). As Stephanos began to achieve stabilization, and to use the new skills and strategies, his reactivity reduced and symptoms improved. Subsequently, he became aware of his own traumatic experiences and was slowly able to name some of his traumatic memories. It was at this time (about 3 months into treatment) that Stephanos was first able to complete a drawing of himself.

Gradually, adverse childhood experiences surfaced and were shared by Stephanos in symbolic (drawings) and straightforward, detailed reporting as the therapeutic relationship strengthened. There was gradual disclosure of the great amount of neglect, physical 
abuse, beatings, chained to the roof, the emotional abuse, and the sexual abuse that he had begun to disclose. He also suddenly disclosed being raped by his father and feeling humiliated and shameful. He had never reported these incidents before.

The standard protocol of EMDR therapy was used to reprocess three specific traumatic memories (using EMDR Phases 3-8). The traumatic memories were (a) father holding a gun to his head (negative cognition $[\mathrm{NC}]$ : "I am in danger"; positive cognition $[\mathrm{PC}]$ : "I can manage"; SUD = 10; initial VOC = 3), (b) being raped by his father (NC: "I am helpless"; PC: "I am ok"; $\mathrm{SUD}=10$; initial $\mathrm{VOC}=2$ ), and (c) being put in a neighborhood garbage bin on wheels and pushed down a hill by his father (details described in the following text).

An example of this reprocessing went as follows: The specific event/target was when his father put him violently in a neighborhood garbage bin on wheels and rolled him down a hill. The most vivid moment was when he slammed into a pole and was injured. The NC was "I am dying," whereas the PC was "I can handle it." The VOC for the PC was initially at 3. He identified feeling anger and fear and reported a score of 10 on the SUD scale. Stephanos located the disturbance in his chest. After seven sets of bilateral stimulation (BLS), which was faster than we expected, he reported a SUD score of 0 . The PC was installed with a VOC of 7 , and the body scan at session end was clear of disturbance. The "conference table" technique was used in the beginning of the session and the EPs were cooperative and in agreement to help Stephanos reprocess the particular traumatic memory.

The other two memories treated with EMDR were also reprocessed effectively. SUD ratings were reduced to 0 and the VOC for each was 7. Parallel to this, Stephanos's behavior began to improve on the ward where he was monitored daily by staff.

Phase III-Integration of Traumatic Memories Into Life Narrative and Continued Rehabilitation. After 3 months of working with Stephanos, he slowly began reporting his history in more detail. He began to build a more coherent narrative of his life that included the realization of these traumatic events. It was still difficult for him to arrange events in chronological order. Trauma had obviously affected his orientation to time. There were moments when he relived the past in the present and other times when he was overwhelmed by emotion and unable to put things in order. We understood these moments as switches into EPs. Stabilization enabled him to tolerate affect and eventually to reprocess.

Over time, personification and presentification increased and he was able to report what happened to him in the past. Stephanos began to recognize and accept his
EPs well, before traumatic memories were reprocessed. Personification and awareness of EPs occurred during and after the stabilization phase and increased throughout treatment. He began to make sense of the senseless and began to integrate the pieces of his life. He was able to relate his story as pertaining to past events and he looked toward the future for life options.

The time restraints of the setting did not allow the therapists to reprocess all targets identified in terms of past events, present triggers, and future concerns. The therapists were concerned with building a relationship with the adolescent, seeing him become more connected to his own resources and developing new resources while focusing on past traumatic events as they were shared during the process. The therapists received the information that the adolescent would be leaving the hospital a few weeks prior to his transfer; therefore, they focused on consolidating what had been done so far and providing a proper closure to the entire body of work. It was the desire of the therapists to continue the work and complete all reprocessing of past, present, and future targets, but it was not clear that this would be possible at the time of closure.

\section{Results}

\section{Outcome and Results of Assessment Battery} and Drawings

Overall, the results of the assessment battery are summarized in Figures 2 and 3, which depict the changes in Stephanos's scores (i.e., UCLA PTSD-RI, SDQ-HEL, SCARED-SF, S-anxiety Inventory, IES-R, DES-II, OSS-3) or frequency of behavior (i.e., O'Neill's Checklist) on each of the assessment tools from baseline to the end of treatment.

UCLA PTSD Reaction Index. At baseline, Stephanos's score was indicative of the presence of PTSD symptoms, although this was not the case at the end of treatment. The percent decrease from Stephanos's index at baseline to the end of treatment was $85 \%$.

Strengths and Difficulties Questionnaire-HEL. The Total Difficulties Score of the SDQ-HEL at baseline indicated the presence of clinically significant problems, although at end of treatment, this was not the case. The percent decrease from Stephanos's Total Difficulties Score at baseline to the end of treatment was $80 \%$.

Screen for Childhood Anxiety Related Emotional Disorders-Short Form. The score for the SCARED-SF at baseline was the highest score possible indicating severe anxiety. The percent decrease from Stephanos's score at baseline to the end of treatment was $60 \%$, indicating that his experience of anxiety decreased by the end of treatment. 


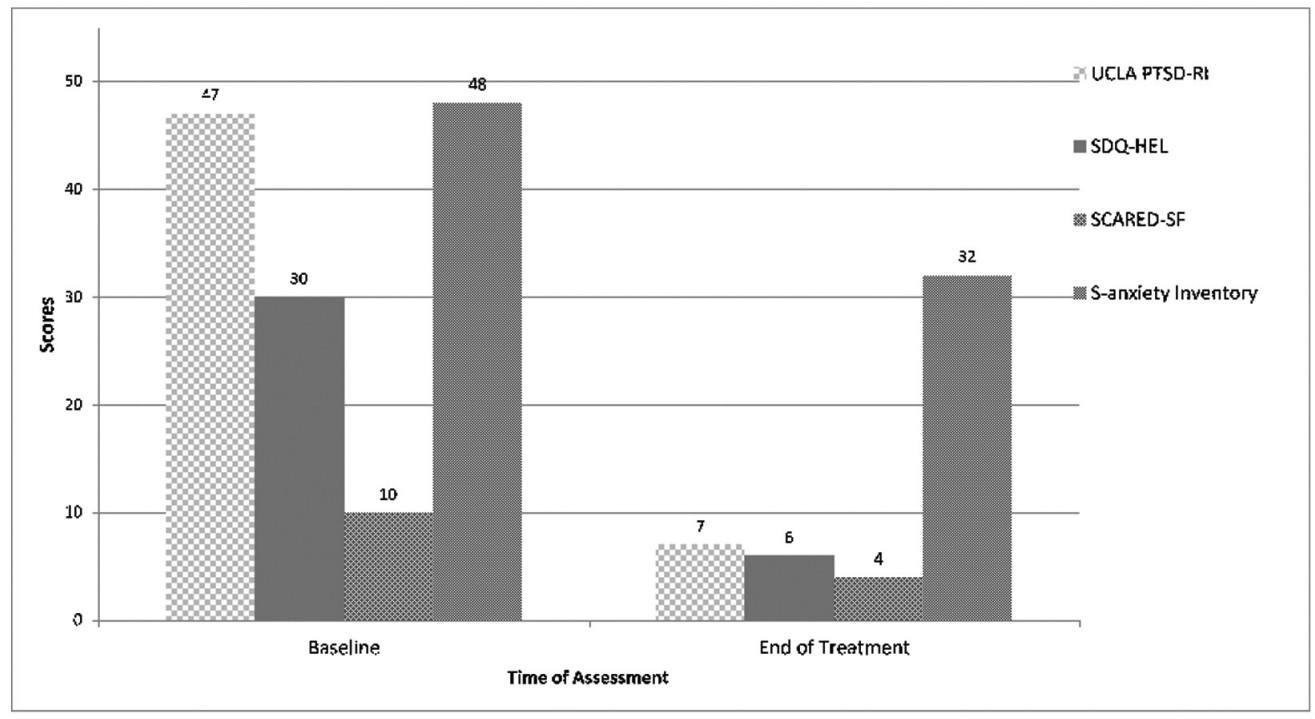

FIGURE 2. Stephanos's scores on the UCLA PTSD Reaction Index (UCLA PTSD-RI), Strengths and Difficulties Questionnaire-HEL (SDQ-HEL), Screen for Childhood Anxiety Related Emotional Disorders-Short Form (SCARED-SF), and State Anxiety (S-anxiety) Inventory at baseline and at the end of treatment.

State Anxiety Inventory. Stephanos's score on the S-anxiety Inventory decreased by $33 \%$, thus showing a decrease in the experience of state anxiety from baseline to the end of treatment.

Impact of Event Scale-Revised. Although both Stephanos's scores at baseline and at the end of treatment were indicative of PTSD symptomatology, the percent decrease from treatment onset to the end of treatment was $21 \%$.

Dissociative Experiences Scale II. Stephanos's score at baseline was indicative of experiences of dissociation that require follow-up, although this was not the case at the end of treatment. The percent decrease was $66 \%$.

Oslo 3-Item Social Support Scale. On the OSS-3, even though the percent decrease of scores was $22 \%$ from baseline to the end of treatment, Stephanos scored high during baseline, throughout treatment, and at the end of treatment indicating that he felt socially supported. At no point did Stephanos score below the cut-off score indicating that he perceived having poor social support.

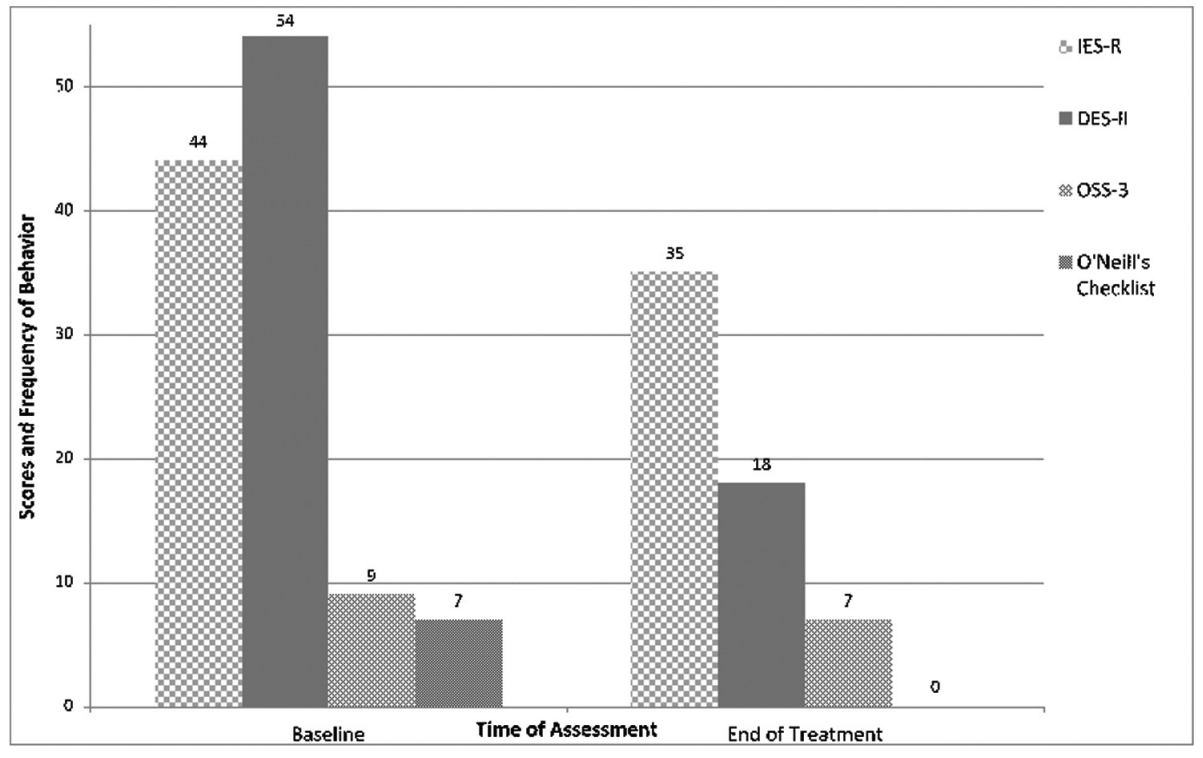

FIGURE 3. Stephanos's scores on the Impact of Event Scale-Revised (IES-R), Dissociative Experiences Scale II (DES-II), Oslo 3-Item Social Support Scale (OSS-3), and frequency of behavior on O'Neill's Checklist at baseline and at the end of treatment. 
O'Neill's Checklist. In addition to providing frequency of targeted behavior, O'Neill's Checklist allows the observer to provide information on the antecedents and consequences of target behaviors. Thus, one may conclude based on these data that when Stephanos was asked or required to do something or when he was asked to engage in a difficult task, he initially yelled and fought to gain attention to avoid doing the task or to avoid a person. O’Neill's Checklist showed a $100 \%$ decrease in the frequency of the three target behaviors (i.e., 1. Hitting; 2. Destroying; 3. Yelling/fighting) recorded daily throughout treatment. In fact, according to the data, these behaviors decreased to zero frequency at the end of treatment.

Drawings. The drawings (Figure 4) were introduced to Stephanos after he had started exhibiting signs of stabilization and being present in the sessions, and it should be noted that it took several months to get Stephanos to cooperate. The first drawing can be considered a baseline drawing. The second drawing was later in therapy. The third drawing was a mandala at the closure of therapy. The fourth drawing was at posttreatment to evaluate if Stephanos maintained his progress at treatment closure approximately a year later.
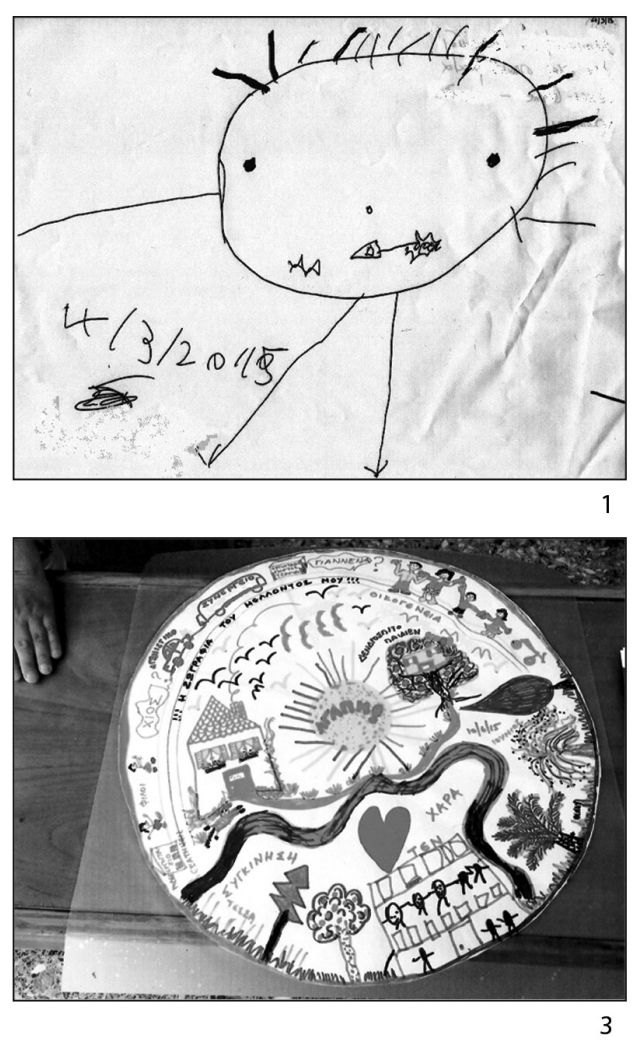

All four of his drawings showed a significant emotional shift. In the first drawing, he described himself as "a jerk, a bad kid without feelings; I hit, I kick, and I kill." In the second drawing, he drew himself with a big red heart and the depiction of his body is clearly more integrated than the previous drawing. The third was a mandala that was created by both therapists and Stephanos over a period of 2 weeks. The reason the mandala was initiated was to express how he was feeling in the present and what his dreams were of the future and to gain closure for the treatment in general. It was a way of saying goodbye. The mandala was laminated and given as a gift during the last session. The fourth drawing was created a year later at treatment followup where his drawing was again integrated, and clearly emotionally positive about his experiences with the two therapists. He wrote on the drawing that he was "happy" to see both therapists and welcomed their support.

\section{Psychopharmacological Changes}

When Stephanos was first admitted to the University Department, he was on three antipsychotic agents, and antiparkinsonian drug and benzodiazepine, which were gradually decreased. When discharged from the University Department, he was on one antidepressant and a small dose of antipsychotic medication.
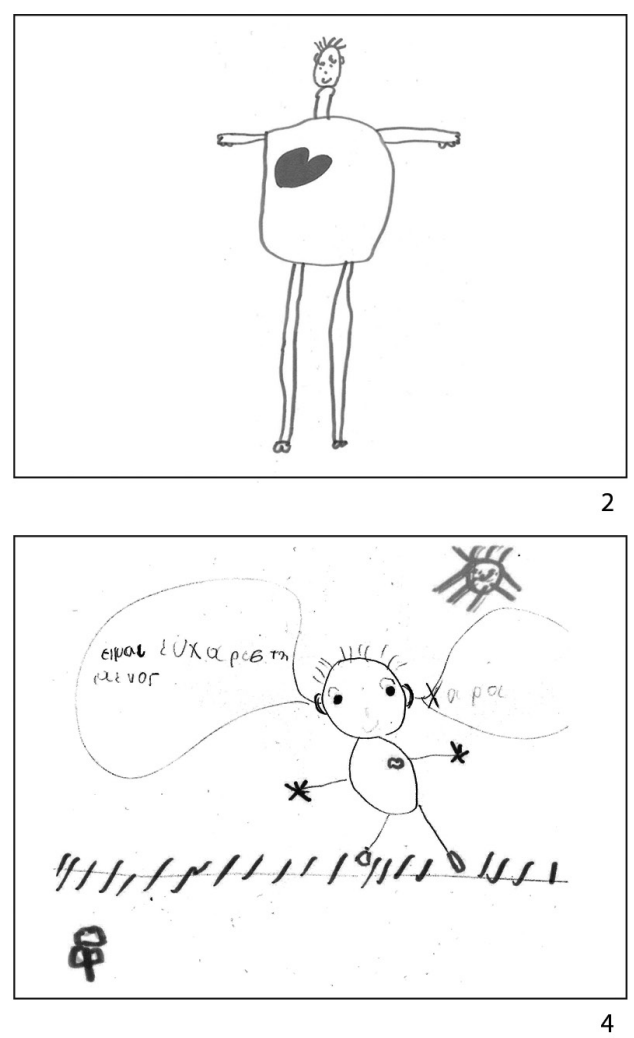

FIGURE 4. 1: First self-image during treatment. 2: Second self-image, more integrated. 3: Mandala- closure of treatment. 4: Follow-up one year. 


\section{Follow-Up}

After being moved to two different residential placements around the country, Stephanos finally settled again in another group home. The two EMDR therapists met with him 11 months later. He communicated quite lucidly, explaining that he was pleased and happy to see them. He remembered a lot of the work he had learned during the therapy together and mentioned that he tried to use it. He lucidly talked about his life and his current living environment and what had happened in his life since he last saw the therapists. He reported positive experiences with staff and coresidents in his group home. In addition to this, his child psychiatrist and his judicial case handler both reported to the therapists that his behavior was well adjusted.

\section{Discussion}

The results indicate that treatment was effective for Stephanos. The objective data, as well as the client's clinical picture as reported by support staff, indicate a significant behavioral change in Stephanos. His overall experience of anxiety decreased over time (as measured by the STAI and the SCARED-SF). In addition, the symptoms of PTSD decreased over time (as measured by the PTSD-RI and IES-R). According to the DES-II scores, the frequency of Stephanos's reported dissociative experiences also decreased. His perception of problems decreased according to the SDQ-HEL. Finally, Stephanos from the onset of treatment, felt that he was in a socially supported environment (according to the OSS-3). Furthermore, Stephanos's maladaptive behaviors of yelling, hitting, fighting, and destroying materials decreased and became nonexistent over time. In addition, changes were also apparent in Stephanos's drawings in that by the end of treatment and at 1-year follow-up, his drawings reflected more integration (Figure 4). Thus, it is fair to conclude that with the receipt of EMDR therapy, combined with the hospital treatment, Stephanos experienced clinically significant improvement of his symptoms and behaviors. In addition, Stephanos reported being and feeling better overall. The staff also reported an improvement in Stephanos especially in the decrease in maladaptive aggressive behaviors (recorded using O'Neill's form).

\section{Treatment Implications}

Integrating TSDP with its understanding of dissociation, and EMDR therapy with its underpinning of AIP case conceptualization, provided an effective intervention for Stephanos. The AIP model viewed the repetitive serious traumatization and institutionalization that he experienced as having led to his pathology. TSDP gave the clinician and the client a foundation for understanding the dissociation of the personality. EMDR therapy was an effective approach to process the dysfunctionally stored memories that were driving symptoms, allowing Stephanos to understand and view his experiences in a more integrated and cohesive manner.

Working with Stephanos proved to be a profound experience for the therapists, which contributed to their already established knowledge and experience regarding the devastating effects of neglect and abuse. The case highlighted important aspects of working with dissociative clients. From a TSDP perspective, the therapists observed how Stephanos's emotional reactions to traumatizing events and situations had been disconnected into different parts of the personality. Although maintenance of this dissociation of the personality appeared to be an adaptive survival strategy, it prevented integration and emotional learning. In addition, the therapists learned that even the most abused have resources and that assisting the client to identify, connect to, and develop these resources was an important part of treatment. It was essential to do this work before using the standard protocol of EMDR for reprocessing traumatic memories. Stabilization, which entailed learning emotional regulation, containment of emotions and thoughts, observing oneself (mindfulness), recognizing and connecting body sensations to corresponding to emotional states while normalizing them (thus decreasing fear and avoidance) was also an important aspect of treatment. Specifically, regarding Stephanos, the therapists discovered that the therapeutic relationships in his life were his "sane" lifelines that kept him grounded and gave him a sense of being in the moment as well as having perspective for the future. In addition, the therapists also learned that understanding and trust can be established and that achieving this helped Stephanos restore his belief in the goodness of human kind. In addition, this experience highlighted that it is in fact possible to help youngsters rebuild a self.

Through the experience of this case, the therapists want to emphasize the importance of mental health practitioners "stepping into the shoes" of the youngsters when working with them and to see and feel the world as they experience it. Furthermore, it is important for mental health practitioners to approach youngsters with a clear and focused framework or concept such as the TSDP. This framework prevented the focus of therapy from being derailed by the client's emotional responses and symptoms; it provided meaning to the symptoms; and allowed for a parallel treatment of the trauma. 
Finally, it was useful to have two therapists working on this case. Both therapists conducted all sessions together. When Stephanos engaged in limit-testing and/or exhibited challenging behaviors to avoid or escape facing the trauma, having a second therapist present to focus on treatment proved to be beneficial. As Stephanos engaged one therapist, the other was able to refocus the session. In addition, it was most helpful to have the opportunity of consultation before and after the sessions.

EMDR therapy and the standard protocol may not be the initial treatment approach for clients who are unable to connect in a trusting relationship with the therapist and who remain in high levels of arousal and disconnection. Although, it should be noted, that in the therapists' experience, there has not been a single traumatized child or adolescent with PTSD that did not benefit from the approach of EMDR therapy and the conceptual understanding of TSDP.

The hospital setting provided a stable and caring environment for Stephanos. He felt connected to the hospital staff. In addition, further advantages included the services that were provided by the hospital (described earlier in this article). All this set the stage for the therapists to parallel the care Stephanos received at the hospital with EMDR therapy. The department administration had invited the therapists to provide this parallel treatment for Stephanos and was supportive of EMDR therapy and the therapists providing it. The disadvantage of the hospital setting was that because it was a short-care unit, Stephanos was discharged prior to completion of EMDR therapy. However, if it were not for the hospital administration and their efforts to keep Stephanos longer, treatment would have been even shorter.

It is unfair to speculate which treatment results can be attributed to the hospital program and which to EMDR without clear data supporting the effects of each. However, it is the opinion of the therapists that EMDR therapy and emphasis on stabilization prior to focusing on processing of the trauma contributed to the decrease of maladaptive behaviors exhibited by Stephanos on the unit.

Finally, incorporating a battery of assessment tools during the course of treatment as measures of cognitive, emotional, and behavioral changes over time was very useful. The assessment tools and monitoring of changes over time were used to obtain data to show treatment results. This is very important for practitioners who live in the clinical world of their practice or agency. Monitoring with objective measures helps clinicians share their results of practiced based work in an evidence-based manner and therefore speak with more clarity about their clinical work with individuals. This work also inspired the wider staff of the hospital to be more sensitive for signs and symptoms of trauma-generated dissociation and to request EMDR therapy more often.

\section{Recommendations}

Ongoing supervision and consultation is recommended for all practitioners invested in treating developmental and trauma-generated dissociation. Furthermore, we encourage all EMDR therapists to incorporate objective and subjective assessment tools throughout their clinical practice to measure their clients' emotional, behavioral, and cognitive changes over time whether the contribution is via randomized controlled trials or via case studies. Both methods contribute to our knowledge base of EMDR therapy.

\section{Limitations}

This is a case study and as such, the results may not be generalizable to other patients. EMDR therapy was provided in conjunction with an inpatient hospital program, and consequently, it is not possible to separate the unique effects of EMDR and the effects of the hospital program. Nonetheless, the data of the case study show clinical significance and improvement in the life of an adolescent at a critical age.

\section{Conclusion}

In this article, we presented a case of an adolescent who had endured prolonged childhood abuse. After 24 sessions of EMDR therapy in an inpatient hospital setting, over the course of 7 months, the adolescent benefited from the treatment provided. The hope was to stop the effects of the "cycle of abuse" that could perpetuate through this adolescent's life. An important piece of work was accomplished during this therapy, and there was hope that the effects of the abuse would stop here.

\section{References}

Artigas, L., Jarero, I., Mauer, M., López Cano, T., \& Alcalá, N. (2000, September). EMDR and traumatic stress after natural disasters: Integrative treatment protocol and the butterfly hug. Poster session presented at the EMDR International Association Annual Conference, Toronto, Ontario, Canada.

Birmaher, B., Brent, D. A., Chiappetta, L., Bridge, J., Monga, S., \& Baugher, M. (1999). Psychometric properties of the Screen for Child Anxiety Related Emotional Disorders (SCARED): A replication study. Journal of the American Academy of Child and Adolescent Psychiatry, 38(10), 1230-1236.

Carlson, E. B., \& Putnam, F. (1993). An update on the Dissociative Experiences Scale. Dissociation, 6(1), 16-27. 
Chen, L., Zhang, G., Hu, M., \& Liang, X. (2015). Eye movement desensitization and reprocessing versus cognitivebehavioral therapy for adult posttraumatic stress disorder: Systematic review and meta-analysis. The Journal of Nervous and Mental Disease, 203(6), 443-451. http: / / dx.doi.org/ 10.1097/ NMD.0000000000000306

Childre, D., \& Rozman, D. (2007). Transforming depression:

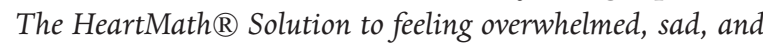
stressed. Oakland, CA: New Harbinger.

Dalgard, O. S. (1996). Community health profile as tool for psychiatric prevention. In D. R. Trent \& C. A. Reed (Eds.), Promotion of mental health (pp. 681-695). Aldershot, United Kingdom: Avebury Press.

Faretta, E. (2013). EMDR and cognitive behavioral therapy in the treatment of panic disorder: A comparison. Journal of EMDR Practice and Research, 7(3), 121-133. http: / / dx.doi.org/10.1891/1933-3196.7.3.121

Farrell, D. (2015). EMDR HAP training manual and case conceptualization. Watsonville, CA: EMDR Institute.

Gauhar, Y. W. M. (2016). The efficacy of EMDR in the treatment of depression. Journal of EMDR Practice and Research, 10(2), 59-69. http: / / dx.doi.org/10.1891/1933-3196 .10 .2 .59

Giannakopoulos, G., Dimitrakaki, C., Papadopoulou, K., Tzavara, C., Kolaitis, G., Ravens-Sieberer, U., Tountas, Y. (2013). Reliability and validity of the Strengths and Difficulties Questionnaire in Greek adolescents and their parents. Health, 5(11), 1774-1783.

Gonzalez, A., \& Mosquera, D. (2012). EMDR and dissociation: The progressive approach. Charleston, SC: Amazon Imprint.

Goodman, R. (1997). The Strengths and Difficulties Questionnaire: A research note. Journal of Child Psychology and Psychiatry, and Allied Disciplines, 38, 581-586.

Goodman, R. (2001). Psychometric properties of the Strengths and Difficulties Questionnaire. Journal of the American Academy of Child and Adolescent Psychiatry, 40(11), 1337-1345.

International Society for the Study of Trauma and Dissociation. (2004). Guidelines for the evaluation and treatment of dissociative symptoms in children and adolescents. Journal of Trauma and Dissociation, 5(3), 119-150.

International Society for the Study of Trauma and Dissociation. (2016). What is dissociation? Retrieved from http: / / www.isst-d.org/?contentID $=76$

Knipe, J. (2014). The EMDR toolbox: Theory and treatment of complex PTSD and dissociation. New York, NY: Springer Publishing.

Liakos, A., \& Giannitsi, S. (1984). Reliability and validity of the modified Greek version of the Spielberger StateTrait Anxiety Inventory. Encephalos, 21, 71-76.

Martin, K. (2016, April). Advanced dissociative table work: What to do after you "set the table." Paper presented at 2nd Summit of Complex Trauma, Dissociative Symptoms \& EMDR Therapy, Austin, TX.

O’Neill, R., Horner, R. H., Albin, R. W., Sprague, J. R., Storey, K., \& Newton, J. S. (1997). Functional assessment and program development for problem behavior: A practical handbook (2nd ed.). Pacific Grove, CA: Brooks/Cole.
O’Shea, K. (2009). EMDR friendly preparation methods for adults and children. In R. Shapiro (Ed.), EMDR Solutions II: For depression, eating disorders, performance, and more (pp. 289-312). New York, NY: Norton.

Shapiro, E. (2007). Clinical Q\&A. 4 Elements. Journal of EMDR Practice and Research, 1(2), 122-124.

Shapiro, F. (2001). Eye movement desensitization and reprocessing (EMDR): Basic principles, protocols, and procedures (2nd ed.). New York, NY: Guilford Press.

Shapiro, F. (2007). EMDR, Adaptive information processing, and case conceptualization. Journal of EMDR Practice and Research, 1(2), 68-87.

Shapiro, F., \& Solomon, R. (2008). EMDR and the adaptive information processing model. Journal of EMDR Practice and Research, 2(4), 315-325.

Spielberger, C. D. (1989). State-Trait Anxiety Inventory: Bibliography (2nd ed.). Palo Alto, CA: Consulting Psychologists Press.

Spielberger, C. D., Gorsuch, R. L., Lushene, R., Vagg, P. R., \& Jacobs, G. A. (1983). Manual for the State-Trait Anxiety Inventory. Palo Alto, CA: Consulting Psychologists Press.

Steinberg, A. M., Brymer, M. J., Kim, S., Briggs, E. C., Ippen, C. G., Ostrowski, S. A., . . Pynoos, R. S. (2013). Psychometric properties of the UCLA PTSD Reaction Index: Part 1. Journal of Traumatic Stress, 26(1), 1-9. http:// dx.doi.org/10.1002/jts. 21780

Van der Hart, O., Groenendijk, M., Gonzalez, A., Mosquera, D., \& Solomon, R. (2013). Dissociation of the personality and EMDR therapy in complex trauma-related disorders: Applications in the stabilization phase. Journal of EMDR Practice and Research, 7(2), 81-94.

Van der Hart, O., Groenendijk, M., Gonzalez, A., Mosquera, D., \& Solomon, R. (2014). Dissociation of the personality and EMDR therapy in complex trauma-related disorders: Applications in phases 2 and 3 treatment. Journal of EMDR Practice and Research, 8(1), 33-48.

Van der Hart, O., Nijenhuis, E. R. S., \& Steele, K. (2005). Dissociation: An insufficiently recognized major feature of complex posttraumatic stress disorder. Journal of Traumatic Stress, 18(5), 413-423.

Van der Hart, O., Nijenhuis, E. R. S., \& Steele, K. (2006). The haunted self: Structural dissociation of the personality and treatment of chronic traumatization. New York, NY: Norton.

Weiss, D. S., \& Marmar, C. R. (1995). The Impact of Event Scale-Revised. In J. P. Wilson \& T. M. Keane (Eds), Assessing psychological trauma and PTSD: A practitioners handbook. New York, NY: Guilford Press.

World Health Organization. (2013). Guidelines for the management of conditions specifically related to stress. Retrieved from http:/ / www.who.int/en/

Correspondence regarding this article should be directed to Penny Papanikolopoulos, PhD, TACT HELLAS - Training and Consultation Today in Hellas, Clinical Director of Psychological Services, 26 KapetanVarda St., Athens 11744, Greece. E-mail: pennypapanikolopoulos@gmail.com 